\title{
De la normatividad hacia la alteridad. Estudio mixto sobre el tratamiento de la diversidad en películas Disney ${ }^{1}$
}

\section{From normativity to otherness. Mixed-method study on the treatment of diversity in Disney films}

\author{
Vicente Monleón ${ }^{2}$ \\ Universitat de València, España \\ Vicente.monleon.94@gmail.com \\ https://orcid.org/0000-0001-8357-1316
}

\section{Resumen:}

La diversidad es condición sine qua non de la propia existencia, no entendida únicamente a nivel físico sino como cualquier diferencia entre personas. Por ello, esta se percibe como la suma de la normatividad y alteridad. Esta realidad se convierte en una de las pretensiones de la escuela actual, especialmente durante la Educación Infantil como periodo educativo en el que se asientan los valores de tolerancia y respeto. No obstante, se cuenta con barreras en cuanto a dicho paradigma inclusivo con elementos como la cultura visual de la imagen que en parte de sus producciones incluye un discurso separatista, clasista y en contra de la diferencia. Un ejemplo concreto radica en la colección "Los clásicos" Disney como conjunto cinematográfico que exalta la normatividad occidental opacando la alteridad, y presentándola

\begin{abstract}
:
Diversity is a sine qua non condition of existence and it should not be only understood on a physical level but also as any difference between people. For this reason, diversity is perceived as the sum of normativity and otherness. The approach of diversity has become one of the aims of present-day schools, especially during Early Childhood Education because this is the period when the values of tolerance and respect are established. However, there are barriers to this inclusive paradigm. One of them comes from the visual culture of images because part of its productions includes a separatist, classist and anti-difference discourse. A concrete example lies in the Disney "classics". This set of films exalts Western regulations, presenting them as the norm and, therefore, overshadowing otherness. To tackle this
\end{abstract}

1 Como referenciar este artículo (How to reference this article):

Monleón, V. (2021). De la normatividad hacia la alteridad. Estudio mixto sobre el tratamiento de la diversidad en películas Disney. Educatio Siglo XXI, 39(3), 83-108. https://doi.org/10.6018/educatio.423291

2 Dirección para correspondencia (Correspondence address):

Vicente Monleón. Universitat de València, Facultat de Magisteri, Departamento: Didáctica de la Expresión Musical, Plástica y Corporal. Av. dels Tarongers, 4, 46022, València. (España) 
como situación normalizada. Como consecuencia, se procede a un estudio de tipo mixto para mostrar los filmes de esta colección que presentan un tratamiento positivo e inclusivo de la alteridad y que sirven por tanto como recurso didáctico para las plantillas docentes de la etapa educativa de Educación Infantil. Con todo, una de las sub-categorías más destacadas en el conjunto analizado es la normalidad con la que se presentan las Necesidades Educativas Especiales y razas diferentes a la blanca occidental.

\section{Palabras clave:}

Cultura visual; cine de animación; Disney; diversidad; alteridad; Educación Infantil. situation, the present mixed-method study focuses on the analysis of those films within the Disney classics which present a positive and inclusive treatment of alterity. These films, therefore, serve as a pedagogical resource for Early Education teaching staff. One of the most prominent sub-categories emerging from the analyzed films is the normality with which special needs and ethnicities different from the white Western one are presented.

\section{Key words:}

Visual culture; animation cinema; Disney, diversity; otherness; Infant Education.

\section{Résumé:}

La diversité est une condition sine qua non de l'existence même, comprise non seulement sur le plan physique mais comme toute différence entre les personnes. Pour cette raison, la diversité est perçue comme la somme de la normativité et de l'altérité. Cette réalité devient l'un des objectifs des écoles d'aujourd'hui, en particulier pendant l'éducation infantile, période éducative au cours de laquelle les valeurs de tolérance et de respect sont établies. Cependant, il existe des barrières à ce paradigme inclusif avec des éléments tels que la culture visuelle de l'image, qui dans certaines de ses productions inclut un discours séparatiste et classiste contre la différence. Un exemple concret est la collection Disney «Classics» en tant qu'ensemble cinématographique qui exalte la normativité occidentale, éclipsant l'altérité et la présentant comme une situation normalisée. Par conséquent, une étude de type mixte est menée pour montrer les films de cette collection qui présentent un traitement positif et inclusif de l'altérité et qui servent donc de ressource pédagogique pour le personnel enseignant de l'éducation infantile. Dans l'ensemble, l'une des sous-catégories les plus remarquables de l'ensemble analysé est la normalité avec laquelle les besoins éducatifs spéciaux et les races autres que les Occidentaux blancs sont présentés.

\section{Mots clés:}

Culture visuelle; film d'animation ; Disney ; diversité ; altérité ; éducation de la petite enfance.

Fecha de recepción: 16-04-2020

Fecha de aceptación: 28-07-2020

\section{Introducción}

La cultura visual se entiende como todo el conjunto de información difundida en una población a través de las imágenes. Estas tienen un potencial sumamente significativo para asentar una ideología, mentalidad, principios, etc., sobre la sociedad que la consume de manera incons- 
ciente en su cotidianidad. De hecho, autores como Alonso-Sanz y Orduña (2013) o Alonso-Sanz y Huerta (2014) manifiestan la repercusión que dicho compendio efectúa sobre el desarrollo del colectivo infantil quienes tienden a nutrirse durante el periodo vital de la infancia con producciones de animación de cine y televisión.

Dentro de este conjunto de productos audiovisuales destacan aqueIlos con la firma Disney. Esta es una compañía que desde sus inicios en los años '20 del s. XX permanece hasta la actualidad, manteniéndose como hegemónica a nivel mundial; superando su competencia en occidente: Pixar (Catmull, 2008) y DreamWorks (Lugo-Lugo y BloodsworthLugo, 2009), así como de Studio Ghibli en Oriente (Cobos, 2001). Esta marca se caracteriza por perpetuar a lo largo de un siglo de existencia mensajes anclados en una sociedad del heteropatriarcado y discriminatorios. Una gran cantidad de estudios se centran en acotar el machismo Disney a través de sus personajes femeninos (Cantillo, 2011 y 2015). Ahora bien, ¿qué espacio queda para la alteridad?, ¿cómo concibe Disney a quienes rompen con la norma establecida, son diversos y por tanto pertenecientes a la otredad?

Las películas animadas producidas por Disney son un potente recurso para introducir el concepto de alteridad (Giovanni, 2007). No obstante, la problemática radica en la manera en que la productora la aborda. Esta se centra en la otredad para criticarla y atacarla, erigiendo la cultura propia occidental como superior al resto. Autores como Digón (2006), Reguillo (2005), Ros (2007) y Giovanni (2007) defienden este pensamiento. Frente a esta mayoría se presenta una minoría que vislumbra el tratamiento positivo de la alteridad en Disney (Guichot y Bono, 2001). "Parece que Disney, al menos paradójicamente, se concentra en otras culturas para construir o al menos reforzar la visión de la identidad de Estados Unidos" (Napier, 2001, p. 472).

Autores como Giovanni (2007) defienden esta occidentalización en la alteridad americana construida por Disney en sus largometrajes de animación. Justificando esta autora su posicionamiento partiendo de productos audiovisuales que emergen de culturas orientales como la china o la árabe, pero que introducen indumentarias, expresiones y alimentación típica del mundo occidental. Generando una necesidad latente en las escuelas de apostar por la multiculturalidad, ya que uno de los problemas actuales en lo que respecta a la alteridad es el racismo (Muñoz, 2000). 
Por ello, según Alegret (1992) se deben focalizar los esfuerzos en pro de una educación no racista, es decir, aquella que no es indiferente ante situaciones de injusticia escolar por cuestión de raza, la que toma acciones y no medidas superficiales, aquella que cambia de objetivos educativos para contribuir positivamente según los derechos universales y promueve investigaciones sobre la problemática indicada para evitar o al menos pormenorizar las desigualdades sociales que se generan (Correa, Martínez-Arbelaiz, Aberasturi-Apraiz y Gutiérrez-Cabello, 2015). Con todo, el marco legal nacional con el que se cuenta (LOE, 2006; LOMCE, 2013) en materia de educación defiende la correcta inclusión en las aulas de cualquier tipo de diversidad desde las primeras etapas educativas, es decir, la Educación Infantil.

\section{Marco empírico}

El objetivo general de esta investigación consiste en mostrar qué largometrajes de la colección cinematográfica "Los clásicos" Disney (19372016) $)^{3}$ presentan un tratamiento positivo e inclusivo de la alteridad.

También, como objetivos secundarios, introducir de una manera directa a través de sus historias, figuras, discursos o imágenes; la polaridad entre normatividad occidental y alteridad para potenciar el tratamiento de la diversidad en las aulas de Educación Infantil. Revisar en dicho conjunto la ideología Disney en cuanto a los polos opuestos destacados. Todo ello, para etiquetar qué productos audiovisuales Disney de este conjunto son significativos para su utilización en las aulas de Educación Infantil como recurso favorecedor de la inclusión de la diferencia.

Para cumplir con dicho propósito se recurre a una metodología mixta (Cedeño, 2012; Hernández, Fernández y Baptista, 2018) entendida como aquella que combina procedimientos tanto de la cuantitativa (Ramírez y Zwerg-Villegas, 2012; Carbajosa, 2011) como de la cualitativa (Martínez, 2006; Erazo-Jiménez, 2011).Según Hernández, Fernández y Baptista (2018) los Ilamados multi-métodos, métodos mixtos o triangulación metodológica:

3 https://coleccionesclasicas.com/lista-completa-clasicos-disney/ 
[‥] representan el más alto grado de integración o combinación entre los enfoques cualitativo y cuantitativo. Ambos se entremezclan o combinan en todo el proceso de investigación, o, al menos, en la mayoría de sus etapas (...) agrega complejidad al diseño de estudio; pero contempla todas las ventajas de cada uno de los enfoques. (p. 21)

En una primera fase, se visionan los largometrajes de la colección - un total de 60 producidos entre 1937 y 2016 e incluidos en dicho conjunto por basarse en su mayoría en obras literarias clásicas - para establecer de una manera general qué cantidad de películas introducen la temática de la normatividad/alteridad (esta información se refleja en la tabla 2). Tras ello, se etiqueta un total de 48 largometrajes en los que se presenta de manera genérica mostrando una tendencia Disney al respecto pero con ausencia de ejemplos concretos. Los 12 filmes restantes son aquellos que se analizan detalladamente en base a 6 categorías y dos sub-categorías para cada una de estas. Todas estas se codifican para el análisis cualitativo de las variables en la tabla 1. Durante este proceso se analizan, las palabras, los mensajes y discursos recreados en las historias, como elementos de estudio en una investigación cualitativa (Cea, 2001) así como también las imágenes en base a lo establecido por la IBI o Investigación Basada en Imágenes (Alonso-Sanz, 2013; Eisner y Barone, 2006). Los recursos cualitativos utilizados son la categorización y codificación de las variables (Strauss y Corbin, 2002). Para este estudio no se precisa de la utilización de ningún programa-software de análisis de datos mixtos.

Concretamente, los filmes en los que se centra la atención son: Saludos amigos (Disney, 1942), Tiempo de melodía (Disney, 1948), Alicia en el país de las maravillas (Disney, 1951), Lo mejor de Winnie the Pooh (Disney, 1977), Taron y el caldero mágico (Hale, 1985), Pocahontas (Pentecost, 1995), El jorobado de Notre Dame (Hahn, 1996), Tarzán (Arnold, 1999), Fantasía 2000 (Disney y Ernst, 2000), Dinosaurio (Marsden, 2000), Lilo y Stitch (Spencer, 2002), La gran película de Piglet (Pappalardo-Robinson, 2003), Hermano oso (Williams, 2003), Chicken Little (Fullmer, 2005), Descubriendo a los Robinson (Lasseter, 2007), Bolt (Spencer, 2008), Winnie the Pooh (del Vecho y Spencer, 2011) y Zootrópolis (Spencer, 2015).

A continuación, se presenta la Tabla 1 en la que se recogen las 6 variables de estudio relacionadas con sus 6 categorías y con sus sub- 
categorías correspondientes; a las que se les atribuye una determinada codificación.

Tabla 1

Codificación de las categorías y sub-categorías de análisis

\begin{tabular}{clcc}
\hline Codificación 1 & \multicolumn{1}{c}{ Categorías } & Codificación 2 & Sub-categorías \\
\hline V1 & Variable 1. Nivel económico & $\mathrm{R}$ & Riqueza \\
\cline { 3 - 4 } & & $\mathrm{P}$ & Pobreza \\
\cline { 3 - 4 } V2 & Variable 2. Raza & $\mathrm{N}$ & Normativa \\
\cline { 3 - 4 } & & $\mathrm{D}$ & Diversa \\
\cline { 3 - 4 } V3 & Variable 3. NEE (Necesidades & + & Positivo \\
\cline { 3 - 4 } & Educativas Especiales) & - & Negativo \\
\hline V4 & $\begin{array}{l}\text { Variable 4. Conflicto bélico } \\
\text { (oposición de 2 bandos) }\end{array}$ & $\mathrm{M}$ & Maldad \\
\hline V5 & Variable 5. Reinos ambientales & $\mathrm{A}$ & Bondad \\
\cline { 3 - 4 } & & $\mathrm{H}$ & Animal \\
\hline V6 & Variable 6. Tipos de animales & $\mathrm{D}$ & Depredadores \\
\cline { 3 - 4 } & (alimentación) & PR & Presas \\
\hline
\end{tabular}

Finalmente, se etiquetan numéricamente los hallazgos y porcentajes relativos para cada una de estas categorías y sub-categorías, recurriendo así a instrumentos propios de la investigación cuantitativa como las gráficas de barras (Garcés, 2000) en las que se presenta de una manera visual y numérica los resultados de la investigación.

\section{Resultados y discusión}

Los primeros resultados de esta investigación sirven para establecer que películas de esta colección introducen de manera directa el concepto de normatividad y/o diversidad. Así se recoge en la Tabla 2. Posteriormente, dichos hallazgos de muestran de una manera visual y cuantitativa a través de una gráfica de barras presentada en la Figura 1. 
Tabla 2

Relación de películas de la colección "Los clásicos" Disney (1937-2016) defensoras de la normatividad occidental frente a la diversidad

\begin{tabular}{lll}
\hline Películas & Normatividad & Diversidad \\
\hline Clásico 1 \\
Clásico 2 \\
Clásico 3 \\
Clásico 4 \\
Clásico 5 \\
Clásico 6 \\
Clásico 7 \\
Clásico 8 \\
Clásico 9 \\
Clásico 10 \\
Clásico 11 \\
Clásico 12 \\
Clásico 13 \\
Clásico 14 \\
Clásico 15 \\
Clásico 16 \\
Clásico 17 \\
Clásico 18 \\
Clásico Honorífico 1 \\
Clásico 19 \\
Clásico 20 \\
Clásico Honorífico 2 \\
Clásico 21 \\
Clásico 22 \\
Clásico 23 \\
Clásico 24 \\
Clásico 25 \\
Clásico 26 \\
Clásico 27 \\
Clásico 28 \\
Clásico 29 \\
Clásico 30 \\
Clásico 31 \\
Clásico 32 \\
Clásico 33 \\
Clásico 34 \\
Clásico 35 \\
\hline
\end{tabular}




\begin{tabular}{|c|c|c|}
\hline Películas & Normatividad & Diversidad \\
\hline Clásico 36 & & \\
\hline Clásico 37 & & \\
\hline Clásico 38 & & \\
\hline Clásico 39 & & \\
\hline Clásico 40 & & \\
\hline Clásico 41 & & \\
\hline Clásico 42 & & \\
\hline Clásico 43 & & \\
\hline Clásico 44 & & \\
\hline Clásico 45 & & \\
\hline Clásico 46 & & \\
\hline Clásico 47 & & \\
\hline Clásico 48 & & \\
\hline Clásico 49 & & \\
\hline Clásico 50 & & \\
\hline Clásico 51 & & \\
\hline Clásico 52 & & \\
\hline Clásico 53 & & \\
\hline Clásico 54 & & \\
\hline Clásico 55 & & \\
\hline Clásico 56 & & \\
\hline Clásico 57 & & \\
\hline Clásico 58 & & \\
\hline $\begin{array}{l}\text { Tratamiento general de la normatividad / } \\
\text { alteridad }\end{array}$ & $\begin{array}{c}48 / 60 \\
80 \%\end{array}$ & $\begin{array}{c}12 / 60 \\
20 \%\end{array}$ \\
\hline $\begin{array}{l}\text { Tratamiento específico de la normatividad / } \\
\text { alteridad }\end{array}$ & $\begin{array}{c}6 / 18 \\
33,3 \%\end{array}$ & $\begin{array}{l}12 / 18 \\
66,7 \%\end{array}$ \\
\hline
\end{tabular}




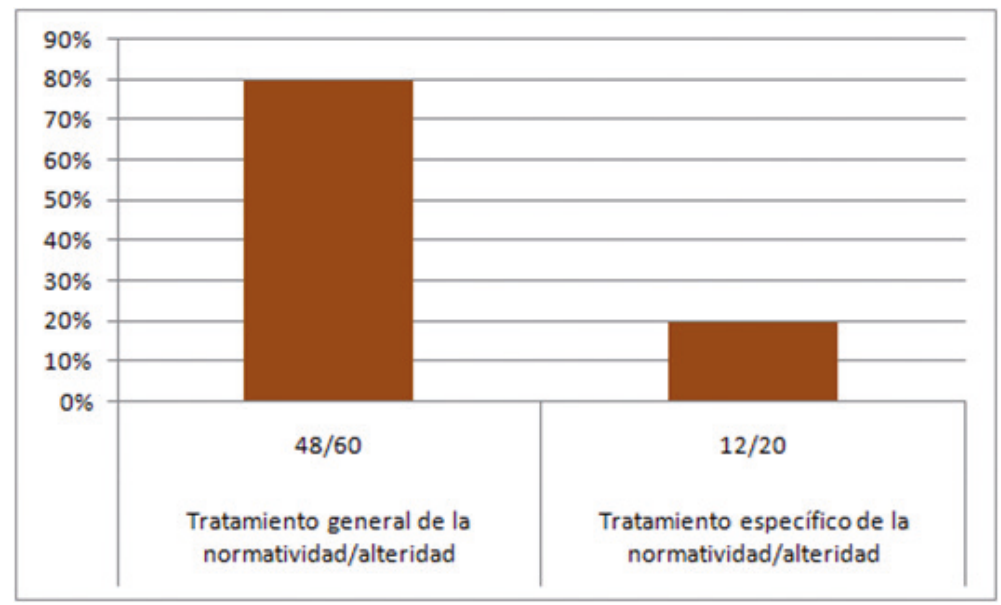

Figura 1. Gráfica sobre el tratamiento general y específico de la normatividad y alteridad en la colección "Los clásicos" Disney (1937-2016).

Tras una primera revisión de largometrajes se etiqueta un total de $48 / 60(80 \%)$ que presentan un tratamiento general de la normatividad/ alteridad, es decir, la defensa de un bando u otro en dichas películas no se manifiesta directamente a través de diálogos, intervenciones o escenas concretas; sino que se efectúa por medio del trasfondo de la trama y la ambientación de la misma. Esta situación conlleva a una asimilación subconsciente y pasiva de una ideología tal y como se defiende desde un posicionamiento de la cultura visual (Torres, 2003; Acaso y Nuere, 2005). Por ello, en materia educativa, se destaca la importancia de analizar críticamente las producciones que el colectivo infantil consume para así evitar la asimilación de unas ideas de manera indirecta y pasiva.

En contraposición, se cuenta con 12/60 películas en las que se anota un tratamiento específico de la normatividad/alteridad que resultan en la muestra de estudio para esta investigación (estos resultados se marcan en color verde en la tabla 2; el color rojo sirve para recoger los largometrajes en los que se basa el estudio). Groso modo, se destaca que el tratamiento general de la variable de estudio se esfuerza en defender una estructura social normativa y defensora de la población norteamericana; negando la existencia de otros colectivos alterosos y/o pormenorizando su realidad. Esta situación no es arbitraria, sino que se justifica con la versión separatista de la Disney defensora únicamente de la raza blanca (Giovanni, 2007; Digón, 2006; Reguillo, 2005; Ros, 2007). 


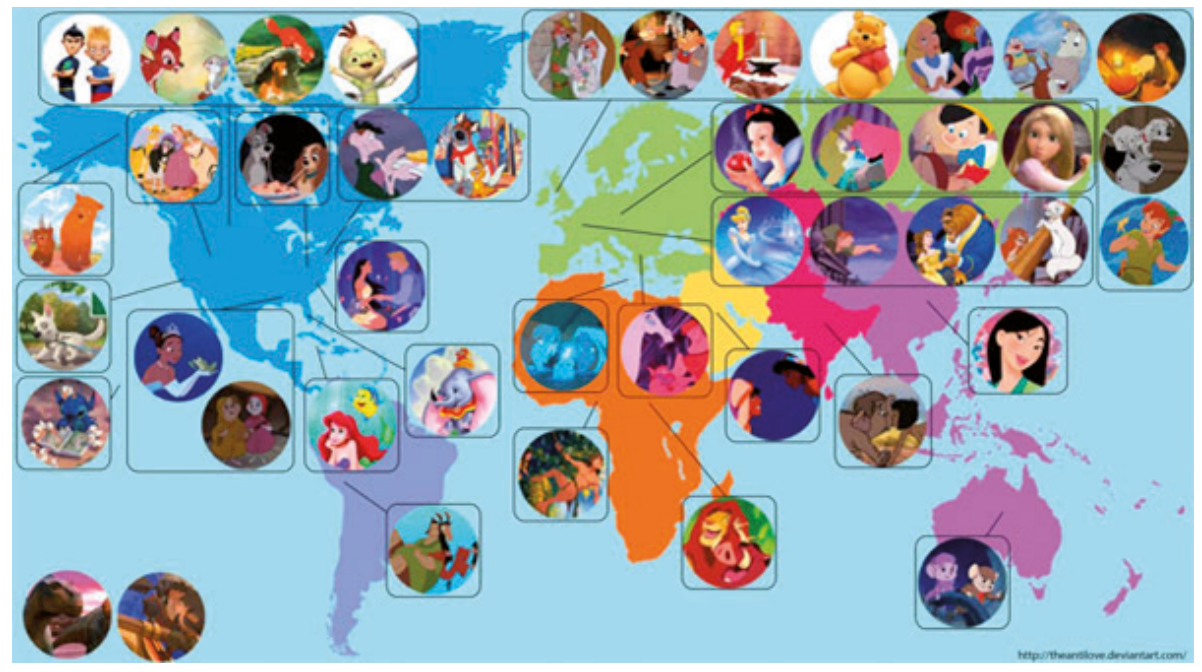

Figura 2. SensaCine (s.f.). Ubicación geográfica de las figuras protagonistas en la colección "Los clásicos" Disney (1937-2016). Recuperado de: http://www. sensacine.com/noticias/cine/noticia-18535968/.

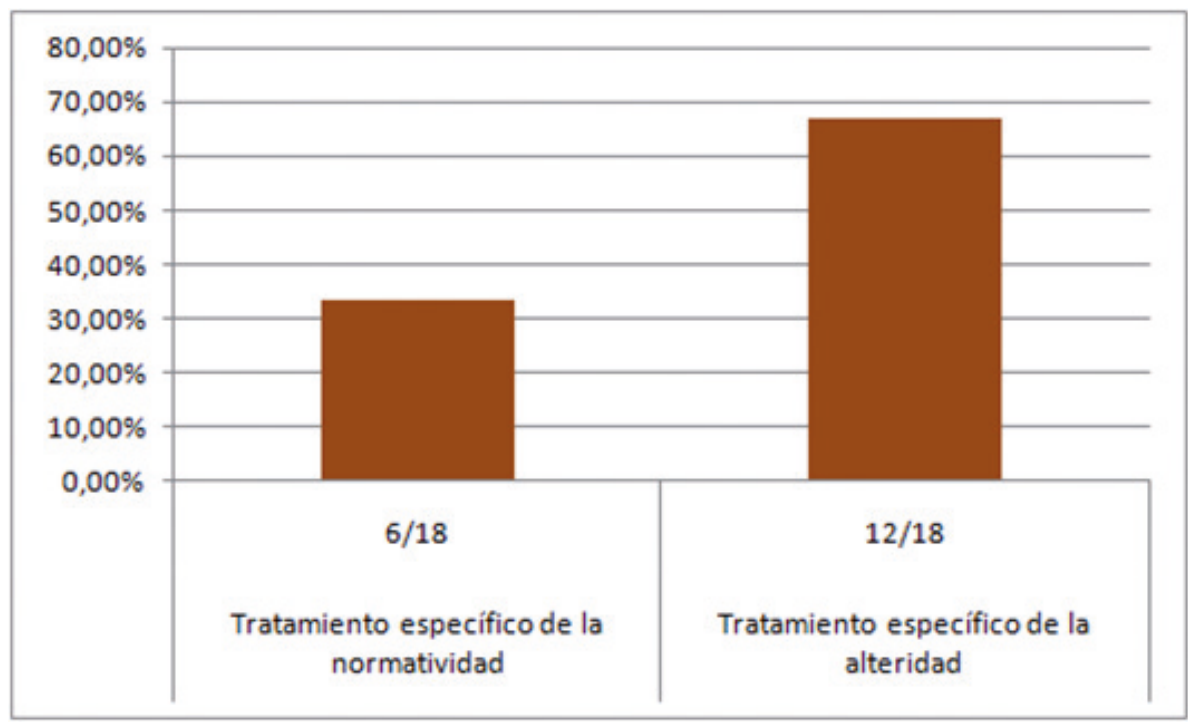

Figura 3. Gráfica sobre el tratamiento específico de la normatividad y alteridad en la colección cinematográfica "Los clásicos" Disney (1927-2016).

Específicamente, en los 12 largometrajes tomados como muestra, se anota una mayoría de 12/18 (66,7\%) situaciones que explicitan una defensa de la alteridad versus una minoría de 6/18 (33,3\%) que apuestan 
por preservar la normatividad occidental como ideal social. Estos resultados manifiestan aquellos filmes que sirven como recursos didácticos para educar en diversidad y en igualdad partiendo de la diferencia en las aulas de Educación Infantil. Concretamente, atendiendo a la tabla 3, se recurre a estos en función de los objetivos que se pretendan trabajar y conseguir en el aula; correspondientes con las 6 categorías y 12 subcategorías. Asimismo, se destaca que la defensa de la alteridad emerge en la colección a medida que esta se prolonga en el tiempo y los intereses sociales cada vez más plurales y globalizados precisan de productos culturales que reflejen dichas realidades diversas; sobre todo impulsadas por las olas feministas (Garabedian, 2014; Chamberlain, 2017).

Tabla 3

Sub-categorías etiquetadas en los largometrajes de la colección "Los clásicos" Disney (1937-2016) en los que aparece un tratamiento directo del concepto alteridad

\begin{tabular}{ll}
\hline PELÍCULAS (MUESTRA) & V1 V2 V3 V4 V5 V6 \\
\cline { 2 - 2 } & R P D D - M B A HDPR \\
\hline
\end{tabular}

Clásico 6. Saludos amigos (Disney, 1942)

Clásico 10. Tiempo de melodía (Disney, 1948)

Clásico 13. Alicia en el país de las maravillas (Disney, 1951)

Clásico 22. Lo mejor de Winnie the Pooh (Disney, 1977)

Clásico 25. Taron y el caldero mágico (Hale, 1985)

Clásico 33. Pocahontas (Pentecost, 1995)

Clásico 34. El jorobado de Notre Dame (Hahn, 1996)

Clásico 37. Tarzán (Arnold, 1999)

Clásico 38. Fantasía 2000 (Disney y Ernst, 2000)

Clásico 39. Dinosaurio (Marsden, 2000)

Clásico 42. Lilo y Stitch (Spencer, 2002)

Clásico 44. La gran película de Piglet (Pappalardo-Robinson, 2003)

Clásico 45. Hermano oso (Williams, 2003)

Clásico 47. Chicken Little (Fullmer, 2005)

Clásico 49. Descubriendo a los Robinson (Lasseter, 2007) 
\begin{tabular}{ll}
\hline PELÍCULAS (MUESTRA) & $\mathrm{V} 1 \mathrm{~V} 2 \mathrm{~V} 3 \mathrm{~V} 4 \mathrm{~V} 5 \mathrm{V6}$ \\
\cline { 2 - 2 } & $\mathrm{R} \mathrm{P} \mathrm{D}+-\mathrm{MBAHDPR}$ \\
\hline
\end{tabular}

Clásico 50. Bolt (Spencer, 2008)

Clásico 53. Winnie the Pooh (del Vecho y

Spencer, 2011)

Clásico 57. Zootrópolis (Spencer, 2015)

\begin{tabular}{llllllllllllc}
\hline HALLAZGOS $(\mathbf{X} / \mathbf{1 8})$ & 1 & 0 & 2 & 4 & 4 & 2 & 1 & 0 & 1 & 0 & 1 & 2 \\
\hline PORCENTAJES $(\%)$ & 5 & 0 & 12 & 22 & 22 & 12 & 5 & 0 & 5 & 0 & 5 & 12 \\
\hline
\end{tabular}

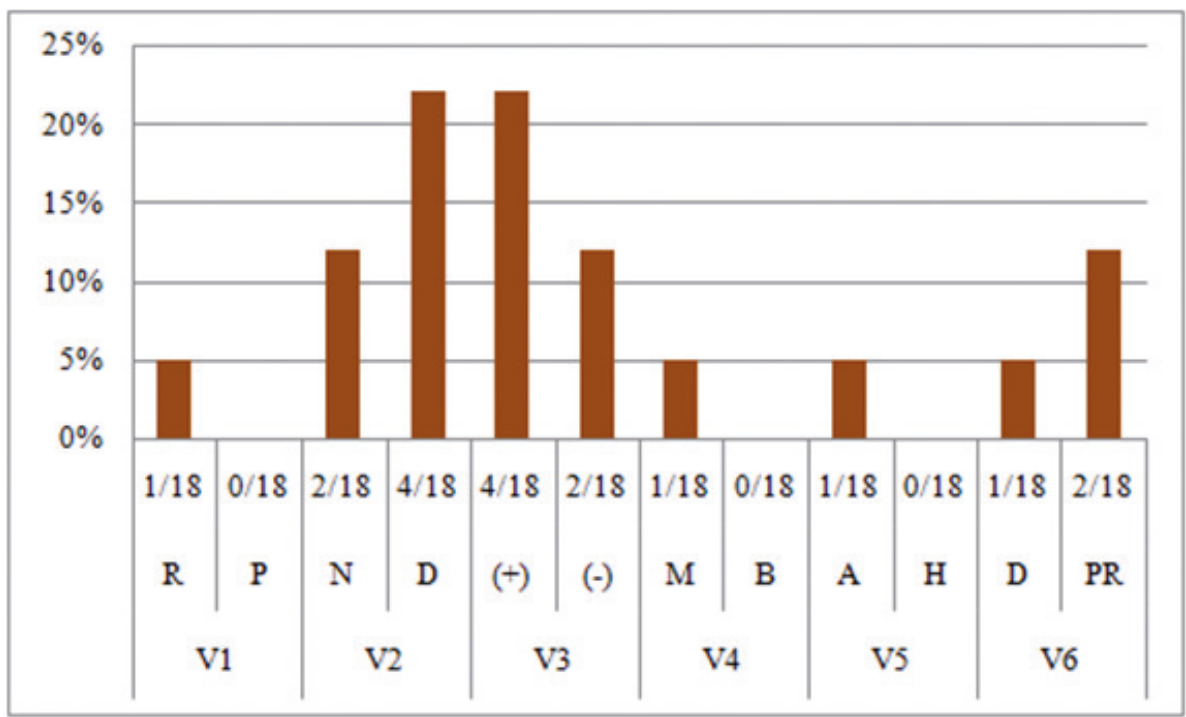

Figura 4. Gráfica de categorías y sub-categorías en la muestra de 18 filmes de la colección "Los clásicos" Disney (1937-2016) que aborda específicamente los conceptos de normatividad y alteridad.

La categoría 1 de este estudio se relaciona con el nivel económico. Esta se subdivide en una visión normativa Disney relacionada con la riqueza correspondiente al 1/18 (5\%) frente a la exaltación de la pobreza como colectivo social alteroso representado con el 0/18 (0\%). Esta situación que dificulta la inclusión de la otredad y la educación en valores de tolerancia y respeto de colectivos infantiles se presenta con el clásico Saludos amigos (Disney, 1942). La película trata sobre un recorrido turístico de Donald junto a Panchito Pistoles y José Carioca por ciudades latinoamericanas, únicamente visibilizando los lugares correspondientes con la riqueza y opacando barrios obreros. 
Estas situaciones deben abordarse en las aulas de una manera normalizada, presentado ambas posibilidades como realidades existentes, sin criticarlas ni juzgarlas; tampoco considerando una inferior frente a la superioridad construida de la otra. Esta es una solución para el tratamiento inclusivo de la diversidad en la escuela. Rompiendo así con el mensaje separatista Disney respecto a las diferencias económicas entre quienes pertenecen a una población (Monleón 2020a y 2020b).

La segunda categoría se corresponde con la raza, diferenciándose en Disney una normativa (12\%) frente a una diversa (22\%). De hecho, resulta significativo para la aplicación didáctica en las aulas de Educación Infantil que una mayoría de los largometrajes utilizados introduzca específicamente otras culturas ya que así se contribuye a una normalización de la multiplicidad de estas. De hecho, la literatura especializada (Bernabé, 2012; Roig, 2012) manifiesta la importancia del tratamiento de la multiculturalidad.

En esta muestra estudiada se etiquetan dos ejemplos que defienden directamente la población blanca y la construyen con superioridad al resto. Por un lado, se destaca el cortometraje "Juanito manzanas" perteneciente al filme Tiempo de melodía (Disney, 1948) en el que se presenta una visión histórica sesgada ya que una de las escenas refleja la convivencia pacífica de la población indígena norteamericana junto a los colonos ingleses, idealizando los primeros frente a los segundos. Por otro lado, en Alicia en el país de las maravillas (Disney, 1951) se rescata esta idea de superioridad en la raza blanca y de victoria tras la conquista de América. Una vez más se cuenta la historia desde el paradigma del grupo vencedor, obviando la realidad de la alteridad vencida. De hecho, por medio de este discurso se manifiesta en el largometraje: "Tras una navegación de más de dos meses por mares desconocidos, en la gloriosa mañana del 12 de octubre descubrió el nuevo mundo Cristóbal Colón. Ese acontecimiento conmovió profundamente a Europa dando a Colón la gloria de su inmortal hazaña que fue premiada por la Corona de España".

Con este ejemplo se refleja el potencial que tiene la imagen para transmitir una deformación de la realidad de manera sesgada (Aguaded, 2015). Por ello, se anima desde la Educación Infantil a introducir una alfabetización audiovisual crítica que ayuda al colectivo de menores a desarrollarse integralmente; cuestionando la información que la cultura visual le impone de manera subversiva (Monleón, 2020a). 


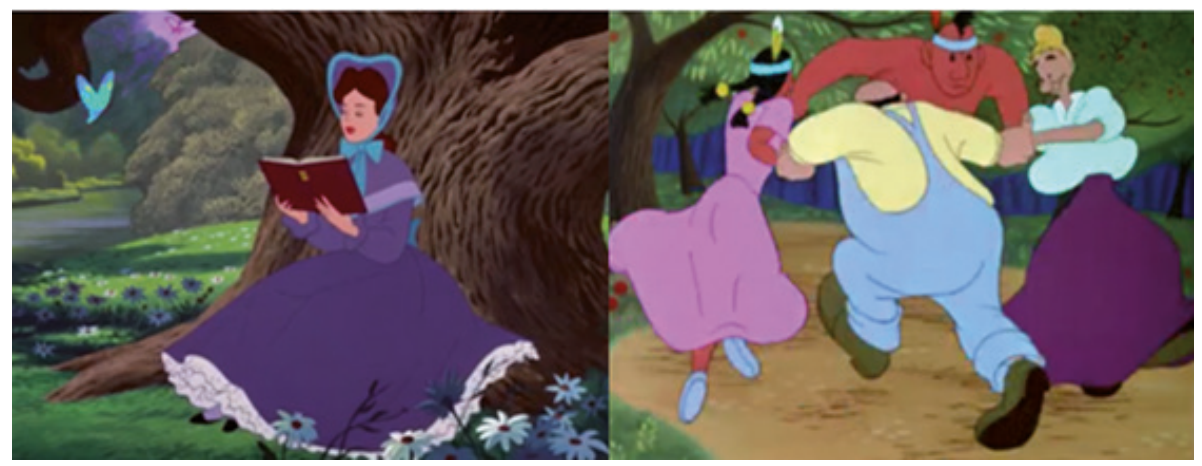

Figura 5. Defensa de la normatividad occidental en Alicia en el país de las maravillas (Disney, 1959) y sesgo histórico-social en Tiempo de melodía (Disney, 1948).

En contraposición, se presentan 4 ejemplos concretos que sirven para aceptar la alteridad y la diferencia como condición explícita de la propia existencia. Es necesario visibilizar dichos grupos sociales y reconocerlos en primera persona tal y como se plantea en Pocahontas (Pentecost, 1995). Este largometraje diseña como figura principal a una mujer perteneciente a la población indígena norteamericana mientras que el conjunto de figuras perversas se destina para la normatividad occidental y la población blanca. Este producto audiovisual resulta interesante para promover la inclusión en las aulas de Educación Infantil ya que cuestiona la normatividad a partir de la propia alteridad que se visibiliza y empodera. De hecho, este es el primer ejemplo cinematográfico Disney de la colección que invierte la concepción de normatividad-alteridad.

Asimismo, con Lilo y Stitch (Spencer, 2002) se replantea la normatividad y la existencia desde el paradigma de la alteridad. Este largometraje se centra en una trama protagonizada por miembros de la humanidad y alienígenas; así como también en el proceso de conocimiento entre ambas poblaciones. El protagonismo reside en quienes habitan en el espacio, por ello, se difiere una visión de las personas como alterosas y satíricas: "son criaturas extremadamente simples" e "¿inteligentes?, no, pero son muy delicados; cada vez que un asteroide choca contra el planeta tienen que empezar la vida desde cero". Estos dos ejemplos sirven para iniciar con grupos de Educación Infantil en la auto-crítica y en la reflexión sobre qué se entiende por normatividad y alteridad; difundiendo así una idea de diferencia en la primera cuando es contemplada desde la segunda. De hecho, autores como Aparici, Escaño y García-Marín 
(2018) o Flecha y Villarejo (2015) defienden una educación crítica desde los primeros niveles educativos. Un acercamiento similar se presenta también con Chicken Little (Fullmer, 2005) película en la que se combina la humanidad con la población espacial ficticia.

Finalmente, con Tarzán (Arnold, 1999) se introduce el continente africano como lugar geográfico para desarrollar la trama. De hecho, es una ejemplificación a través de la que se aborda específicamente la situación de la alteridad con respecto a la normatividad y de las diferencias y similitudes entre ambos grupos. Esta relación se materializa con la historia de vida de Tarzán y su familia de monos/as.

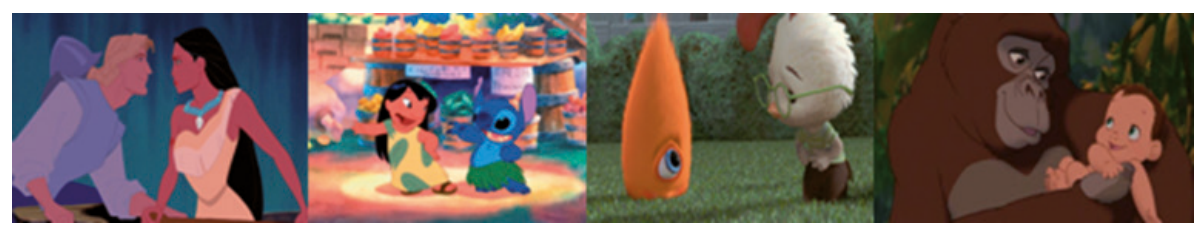

Figura 6. Alteridad entendida como razas diferentes a la blanca y normativa en Pocahontas (Pentecost, 1995), Lilo y Stitch (Spencer, 2002), Chicken Little (Fullmer, 2005) y Tarzán (Arnold, 1999).

La siguiente variable se relaciona con las Necesidades Educativas Especiales. Actualmente, cada vez es más común encontrar diversidad en un aula así como también dificultades en el aprendizaje (Miñambres y Jové, 2000; López, 2011). Por ello, se considera necesario especificar ejemplos concretos de la colección "Los clásicos" Disney para utilizarlos como referencia en las aulas de Educación Infantil, educando así a miembros de la infancia con una mayor tolerancia y respeto respecto a la alteridad.

Los ejemplos más significativos se relacionan con las producciones de Lo mejor de Winnie the Pooh (Disney, 1977), La gran película de Piglet (Pappalardo-Robinson, 2004) y Winnie the Pooh (del Vecho y Spencer, 2011) ya que recogen personajes representativos de trastornos psicológicos.

Winnie presenta un desorden alimenticio, por ello resulta insaciable en cuanto a su alimentación y adicción a la miel. Igor aparece sumido en una depresión continua razón debido a la que se le imposibilitan sentimientos y emociones relacionadas con la alegría. Piglet padece ansiedad ante situaciones novedosas que escapan a su control. Tigger tiene un trastorno por déficit de atención e hiperactividad que se materiali- 
za en el audiovisual por medio de los saltos que efectúa con su cola. Conejo presenta un Trastorno Obsesivo Compulsivo respecto al orden. Christopher Robin padece de esquizofrenia ejemplificada en la relación que cree real con sus juguetes de peluche. Asimismo, búho es un animal narcisista, ya que es incapaz de centrar la atención en otro ser más allá de su propia persona.

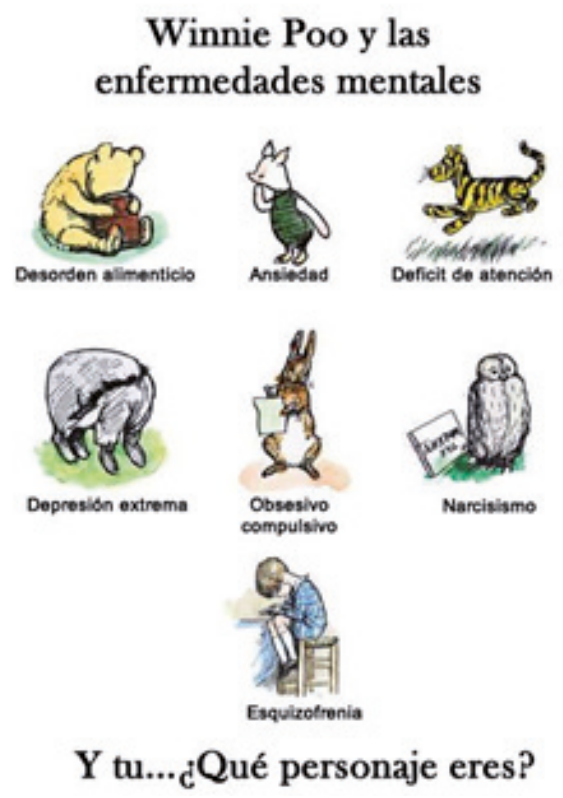

Figura 7. Pinterest (s.f.). Desórdenes en figuras de las historias de Winnie Pooh. Recuperado de: https://www.pinterest.es/pin/470485492297521353/.

Estas diversidades psicológicas que son difundidas a través de personajes de animación Disney sirven como recurso e hilo conductor para introducir la temática en las aulas de Educación Infantil de una manera motivadora y significativa que contribuya a la inclusión real de la infancia alterosa y diversa en la normatividad construida socialmente.

De hecho, el largometraje Fantasía 2000 (Disney y Ernst, 2000) también resulta positivo para ello, ya que en dos de sus cortometrajes se presenta como estandarizada la diversidad a nivel físico. Por un lado, a través del soldadito de plomo carente de una pierna en el corto "Concierto para piano $\mathrm{n}^{\circ} 2^{\prime \prime}$ y del flamenco con diferente color en el plumaje respecto a sus iguales en el corto "El carnaval de los animales". Huerta, 
Alonso-Sanz y Ramon (2019) abordan precisamente con su trabajo las posibilidades que guarda el cine como recurso para educar en diversidad. De hecho, la diversidad se entiende como todo aquello que rompe con la norma establecida y que, por tanto, se convierte en su alteridad.

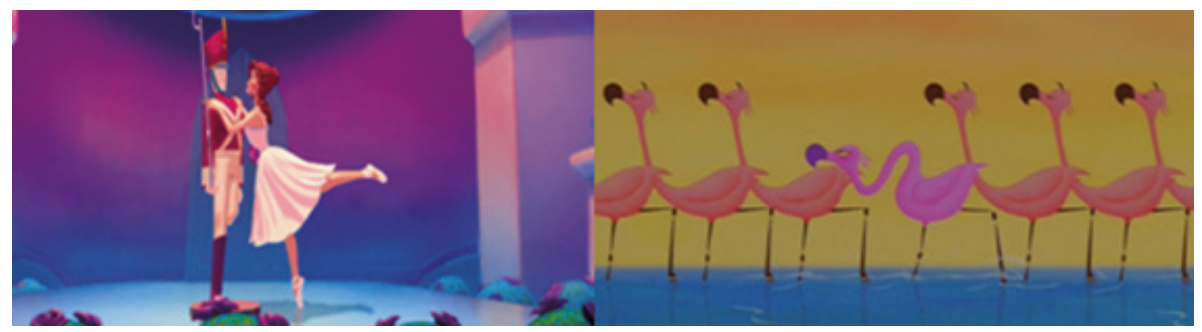

Figura 8. Diversidad física en Fantasía 2000 (Disney y Ernst, 2000).

No obstante, se advierten 2/18 (12\%) ejemplos en los que se atenta de manera directa contra la diversidad. Por un lado, el largometraje El jorobado de Notre Dame (Hahn, 1996). Este trata sobre Quasimodo un niño gitano con imperfecciones que es cuidado por Frollo siguiendo las indicaciones de arichidiácono de la catedral. Este joven permanece toda su vida encerrado en el campanario tal y como le indica su cuidador, argumentando en base a su criterio que la normatividad no acepta y arremete contra cualquier tipo de diferencia. De hecho, así lo comparte a través de la siguiente intervención de una de las canciones del filme: "Estás deforme y eres feo y eso son crímenes que el mundo no permite, ¿no lo comprendes ya? Vete y te dirán que eres un monstruo, vete y siempre se reirán de ti. Vas a osar dejarme a mí y consternarme. Ven aquí y pide excusas. Dime gracias. Lo mejor es, ¿lo ves? Estar aquí".

Asimismo, cuando decide salir es arremetido por toda la población parisina quien lo apresa y ridiculiza. Con todo, al final del largometraje este permanece oculto del resto (García-Lago, 2002). Por lo que esta historia no contribuye a la inclusión de la alteridad, sino a su exclusión; cuando se consume de manera pasiva. Esta situación resulta peligrosa para la educación del colectivo infantil ya que les condiciona inconscientemente una ideología separatista y en contra de la diferencia (Monleón, 2020a). Aunque, resulta un material idóneo para generar crítica y reflexión cuando se plantea en el aula para ser trabajado de una manera activa.

Por otro lado, el largometraje Descubriendo a los Robinson (Lasseter, 
2007) presenta la necesidad educativa de las altas capacidades en el protagonista de la trama, Lewis. Este en un niño huérfano con un gran potencial intelectual pero que tiende a ser rechazado por las familias que lo visitan para adoptarle debido a su no normatividad. Esta situación se tilda de peligrosa cuando el filme se consume de una manera pasiva ya que contribuye a que se genere un rechazo hacia la intelectualidad que sobrepasa los parámetros establecidos como normales. De hecho, es necesario reflejar el olvido que tiende a sufrir dicho colectivo educativo a lo largo de la historia de la educación (Gómez y Mir, 2011).

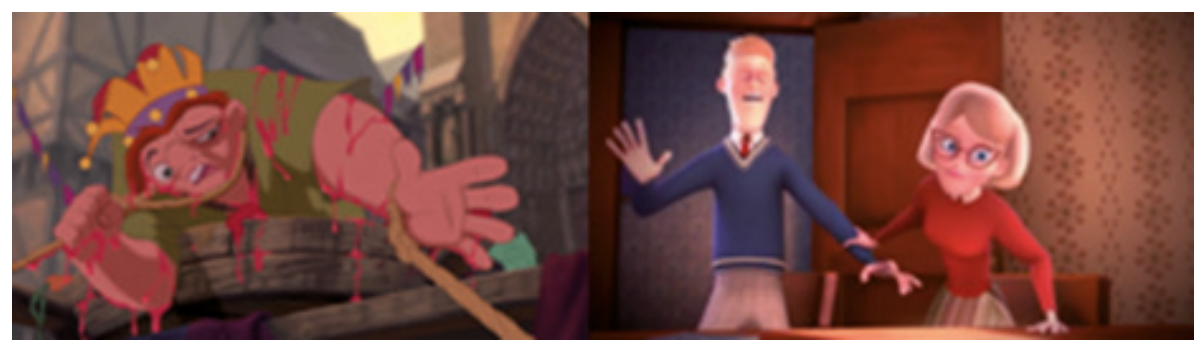

Figura 9. Rechazo a la diversidad en El jorobado de Notre Dame (Hahn, 1996) y Descubriendo a los Robinson (Lasseter, 2007).

La cuarta categoría se relaciona con el conflicto bélico. Esta variable se corresponde con el concepto dual de alteridad/normatividad ya que toda batalla supone el enfrentamiento mínimamente entre dos bandos opuestos que distan en ideología, principios, identidad, etc. Por ello, se rescata para el análisis en este estudio.

Concretamente, como sub-categorías se etiqueta una visión positiva de $0 / 18(0 \%)$ frente a una vertiente que lo presenta como negativa 1/18 (5\%). Esta situación se ejemplifica en el largometraje Taron y el caldero mágico (Hale, 1985), la primera historia Disney de la colección que presenta la guerra como hilo conductor de la misma pero percibida por el conjunto de figuras protagonistas como contraproducente para el progreso social: "la guerra no es un juego, muere la gente" y "el valor imprudente no vencerá la maldad". Por esta razón, se considera un producto cinematográfico de animación útil para introducir en las aulas de Educación Infantil y fomentar el acercamiento entre grupos diferenciados por medio de otros procesos como la palabra. Asimismo, sirve para desmitificar un tema tabú (Cooper, 2002) y favorecer la educación en valores (Guichot-Reina y Merino-Delgado, 2015). 
Le sigue la categoría de los reinos ambientales, centrando la atención en la sub-categoría del reino animal y humano. Estos dos grupos se presentan como opuestos atendiendo a la tendencia social que considera al primero inferior y sublevado al segundo, sin considerar los derechos de vida que presenta partiendo de la mentalidad animalista (Monleón, 2020a).

Concretamente, aparece 1/18 (5\%) ejemplo que defiende al colectivo de animales directamente contra una ausencia de defensa de normatividad en la humanidad 0/18 (0\%). Este se encuentra en el largometraje Hermano oso (Williams, 2003) una historia que versa sobre dichos bandos, sobre el concepto de la propia alteridad y sobre cómo este cambia dependiendo de la posición que se adopte.

Kenai es un joven a quien se le asigna el tótem del oso del amor como principio que debe guiar su vida. No obstante, él odia a dichos animales, los concibe como seres que arremeten contra la humanidad y contra los productos que las personas cultivan. Su odio conlleva al asesinato de uno de estos animales y su transformación en dicho ser como ejercicio de empatía. Esta metamorfosis le contribuye a entender la alteridad desde otro posicionamiento y enseña que lo normativo depende del bando en el que cada persona se encuentre.

Ejemplificándolo con el filme, Kenai apuesta por la normatividad humana cuando este es una persona, pero al ser transformado en animal, su concepción cambia y la normatividad pasa a convertirse en alteridad. Por ello, dicho producto audiovisual se considera idóneo para ser introducido en las aulas de Educación Infantil como un método para favorecer ejercicios de empatía con respecto a la diversidad, la diferencia y la alteridad.

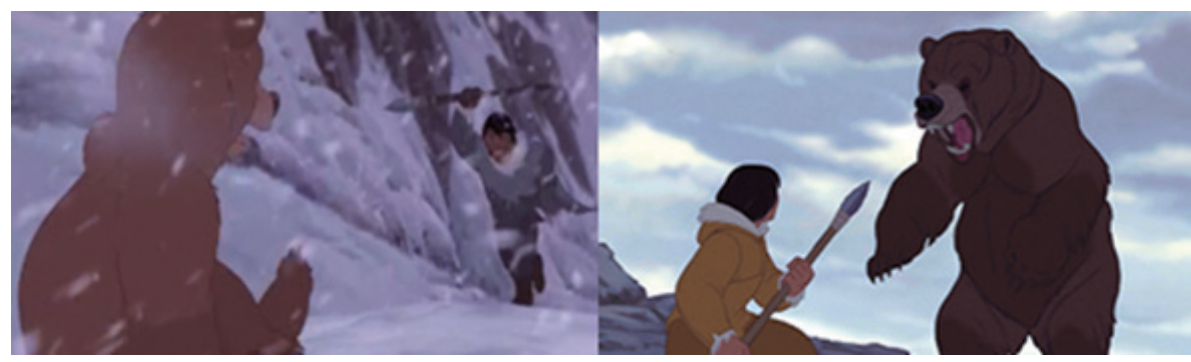

Figura 10. Kenai-oso siendo víctima de la humanidad y Kenai-humano siendo agresor del reino animal en Hermano oso (Williams, 2003).

Finalmente, la sexta variable se centra en el análisis de los tipos de animales clasificados por su alimentación convirtiéndose así en depredadores 
o presas. Esta idea se relaciona con la realidad humana en tanto que se atribuye una normatividad a la superioridad (fortaleza) y una alteridad en cuanto a inferioridad (debilidad). En el caso de los filmes de Disney, se relaciona con la temática de los animales por la cantidad de productos audiovisuales en los que recurre a dicho reino como hilo conductor de las historias, es una de las características esenciales de su animación (Singer, 2018).

Específicamente se anota 1/18 (5\%) de largometrajes en los que perdura una idea de superioridad y normatividad en la especie depredadora y con fuerza. Esta se encuentra en Bolt (Spencer, 2006). La trama se centra en las aventuras de un canino y su cuidadora Penny respecto a la salvación del mundo del doctor Cálico -un hombre que cuenta con un ejército de mininos-. En este ejemplo, la oposición entre bandos queda estereotipada y de manera maniquea con respecto a dichas alteridades; perros/as versus gatos/as.

De manera contrapuesta destacan 2/18 (12\%) ejemplos fílmicos Disney en esta colección que reconocen la alteridad e impulsan la existencia de las especies consideradas como presas. En Dinosaurio (Marsden, 2000) se defiende al grupo de reptiles extinguidos herbívoros, quienes se unen para sobrevivir al ataque de carnívoros (carnotaurus en el film).

En Zootrópolis (Spencer, 2015) se transmite dicha idea a través de la figura de Judy, una coneja que consigue completar su formación y convertirse en la primera presa miembro del cuerpo de policías, hasta el momento una cuadrilla constituida por animales depredadores únicamente. Todas estas situaciones deben ser introducidas en las aulas para desmentir la construcción social normativa y permitir la inclusión en esta de la propia alteridad.

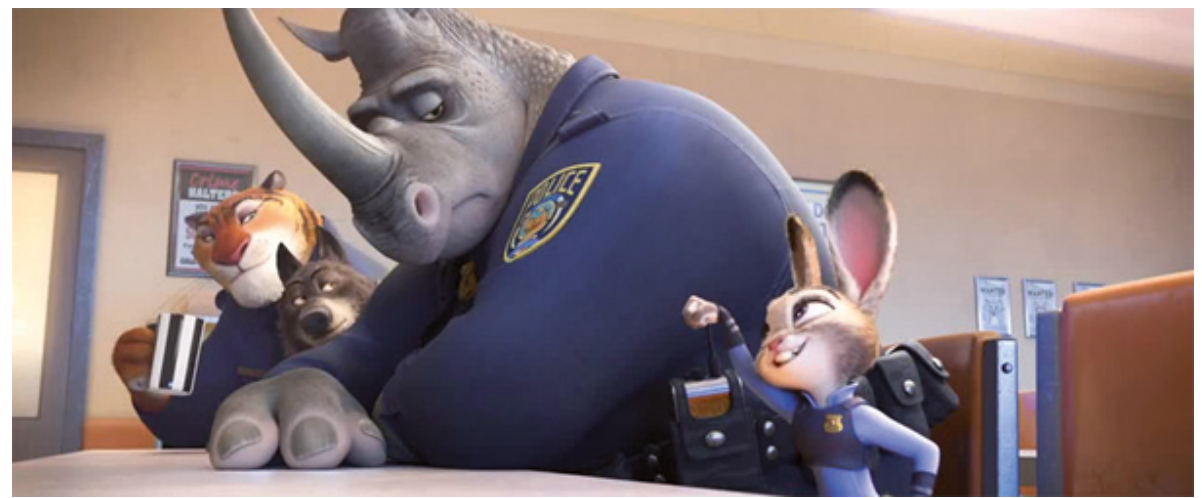

Figura 11. Cuerpo de policía formado por depredadores y la presa Judy en Zootrópolis (Spencer, 2015). 


\section{Conclusiones}

En términos generales, la colección cinematográfica de animación "Los clásicos" Disney (1937-2016) se entiende como un producto de la cultura visual para el colectivo infantil, aunque extrapolable a un abanico poblacional diverso en edad, que tiende a defender la normatividad occidental y a ocultar o ridiculizar a su alteridad, a su opuesto, a quien es diferente y a quien rompe con la norma establecida. Disney utiliza sus productos para generar un imaginario social, mundialmente conocido y reconocido, a pesar de solo incluir y preservar los principios de la sociedad poscolonialista y heteropatriarcal (prototipo de hombre blanco, adinerado, monarca, machista y heterosexual).

Estos mensajes transmitidos con normalidad en dichas películas que tiende a consumir el colectivo infantil desde la pasividad deben ser analizados desde las aulas de Educación Infantil, promoviendo así una educación audiovisual de calidad que sirva para despertar un espíritu crítico y de la sospecha en la ciudadanía. Es necesario posicionarse en el lugar del alter, para reconocerlo, conocerlo y entenderlo. Se precisa de una preparación previa por parte del profesorado, es decir, un consumo activo efectuado por quienes componen las plantillas docentes para desentrañar los mensajes que se difunden con dichos filmes. A este respecto, este artículo contribuye a la alfabetización visual de maestros/as y les ayuda a entender los discursos que difunde Disney y que calan en el subconsciente de la audiencia consumidora de los mismos; sabiendo qué filmes sirven para contribuir a la inclusión de la diversidad en el aula y cuáles dificultan dicho proceso innovador.

Para ello, una propuesta entre una pluralidad de opciones resulta en los discursos empáticos y dramatizaciones en las que se desarrollan roles totalmente opuestos a la propia persona. Por ejemple, quienes se identifiquen como niños deben de asumir la historia de vida de una mujer/hembra del filme y generar empatía con respecto a su desarrollo en la trama; a la inversa con quienes se identifican como niñas. Tras los debates y discursos aflorados durante los periodos de asamblea y puesta en común se propone proceder a dramatizaciones actualizadas de los largometrajes.

Los filmes deben consumirse activamente, por fragmentos, promoviendo actividades previas y posteriores a su visionado. Esta es una de las pretensiones más significativas de la alfabetización audiovisual. De 
esta manera, se enseña a quienes consumen los productos del cine de animación a cuestionarse las ideas que le son impuestas, a valorar la fiabilidad y veracidad en las mismas. El cine como recurso para el entretenimiento y para evadir al alumnado de la realidad durante un tiempo debe desestimarse; sobre todo cuando se recurre a películas que difunden una ideología diferente a la que rige las rutinas del aula y el paradigma socio-educativo de la inclusión.

Con todo, se rescata una serie de productos de este conjunto de películas que sirven para introducir de una manera directa el respeto y tolerancia hacia la alteridad, sobre todo del conjunto de personas con necesidades educativas especiales con las que se conviven en las aulas. Efectuándose siempre de una manera activa, a través de dinámicas, talleres, proyectos de trabajo, actividades de cine-fórum, etc. De esta manera se contribuye a la alfabetización visual de quienes componen la infancia, quienes aprenden a decodificar mensajes transmitidos por el poder a través de sus productos de gran alcance.

Los clásicos que introduce de manera directa el concepto de alteridad son: Lo mejor de Winnie the Pooh (Disney, 1977) para la diversidad funcional y psicológica, Taron y el caldero mágico (Hale, 1985) para el tratamiento de la guerra, Pocahontas (Pentecost, 1995) para la diversidad racial, El jorobado de Notre Dame (Hahn, 1991) para la diversidad étnica y funcional, Tarzán (Arnold, 1999) para la diversidad física, Fantasía 2000 (Disney y Ernst, 2000) para la diversidad funcional y física, Dinosaurio (Marsden, 2000) para la diversidad de edades, Lilo y Stitch (Spencer, 2002) para fomentar la autocrítica, La gran película de Piglet (Pappalardo-Robinson, 2003) para la diversidad funcional y psicológica, Hermano oso (Williams, 2003) para la generación de empatía, Chicken Little (Fullmer, 2005) para trabajar la autocrítica, Descubriendo a los Robinsons (Lasseter, 2007) para la diversidad intelectual, Bolt (Spencer, 2008) para las oposiciones estereotipadas, Winnie the Pooh (del Vecho y Spencer, 2011) para la diversidad funcional y psicológica y Zootrópolis (Spencer, 2015) para las oposiciones estereotipadas. 


\section{Referencias}

Acaso, M. y Nuere, S. (2005). El curriculum oculto visual: aprender a obedecer a través de la imagen. Arte, Individuo y Sociedad, 17, 207-220.

Aguaded, J. I. (2015). Cine, sistema educativo, responsabilidad social e influencia en el entorno ciudadano. Aularia, 2, 9-17.

Alegret, J. L. (1992). Racismo y Educación. En P. Fermoso (Ed.). Educación Intercultural (pp. 93-110). Madrid: Narcea.

Alonso-Sanz, A. (2013). A favor de la Investigación Plural en Educación Artística. Integrando diferentes enfoques metodológicos. Arte, Individuo y Sociedad, 25(1), 111 120. DOI: https://doi.org/10.5209/rev_ARIS.2013.v25.n1.41167

Alonso-Sanz, A. y Huerta, R. (2014). La cultura visual televisiva y las representaciones infantiles. Fotoensayos de un estudio de caso sobre el dibujo en la escuela. Revista SONDA Investigación y Docencia en Artes y Letras, 3, 37-54.

Alonso-Sanz, A. y Orduña, S. (2013). Referentes relativos a la identidad en la Cultura Visual infantil. Aula de Innovación Educativa, 220, 18-24.

Aparici, R., Escaño, J. C. y García-Marín, D. (2018). La otra educación. Pedagogías críticas para el siglo XXI. España: Editorial UNED.

Arnold, B. (productor) y Buck, C. y Lima, K. (directores). (1999). Tarzán [Cinta cinematográfica]. Estados Unidos: Walt Disney Pictures.

Bernabé, M. d. M. (2012). Pluriculturalidad, multiculturalidad e interculturalidad, conocimientos necesarios para la labor docente. Hekademos: revista educativa digital, 11, 67-76.

Cantillo, C. (2011). Análisis de la representación femenina en los medios. El caso de las princesas Disney. Making of: Cuadernos de cine y educación, 78, 51-61.

Cantillo, C. (2015). Del cuento al cine de animación: semiología de una narrativa digital. Revista de Comunicación de la SEECI, 38, 133-145.

Carbajosa, D. (2011). Debate desde paradigmas en la evaluación educativa. Perfiles Educativos, 132(33), 183-191.

Catmull, E. (2008). Cultivar la creatividad colectiva: el sello de Pixar. Harvard Business Review, 86(9), 74-85.

Cea, M. Á. (2001). Metodología cuantitativa. Estrategias y técnicas de investigación social. Madrid: Editorial Síntesis.

Cedeño, N. (2012). La investigación mixta, estrategia andragógica fundamental para fortalecer las capacidades intelectuales superiores. Revista Res Non Verba, 2(2), 17-36.

Chamberlain, P. (2017). Affective Temporalities. En P. Cgamberlain (Ed.). The Feminism Fourth Wave (pp. 73-106). Londres: Palgrave mcmillan.

Cobos, T. L. (2001). La animación japonesa en el contexto occidental. México: Universidad Autónoma de Nuevo León.

Cooper, H. (2002). Didáctica de la historia en la educación infantil y primaria. Ediciones Morata: Madrid.

Correa, J. M., Aberasturi-Apraiz, E. y Gutiérrez-Cabello, A. (2018). Ciudadanía digital, activismo docente y formación de futuras maestras de educación infantil. RELATEC, 15(2), 39-54. DOI https://doi.org/10.17398/1695-288X.15.2.39 
Monleón, V. (2021). De la normatividad hacia la alteridad. Estudio mixto sobre el tratamiento de la diversidad en películas Disney. Educatio Siglo XXI, 39(3), 83-108.

Del Vecho, P. y Spencer, C. (productores) y Anderson, S. y Hall, D. (directores). (2011). Winnie the Pooh [Cinta cinematográfica]. Estados Unidos: Walt Disney Animation Studios.

Digón, P. (2006). El caduco mundo de Disney: propuesta de análisis crítico en la escuela. Comunicar, 26, 163-169.

Disney, R. E. y Ernst, D. W. (productores) y Algar, J., Brizzi, G., Brizzi, P., Butoy, H., Glebas, F., Goldberg, E., Hahn, D. y Hunt, P. (directores). (2000). Fantasía 2000 [Cinta cinematográfica]. Estados Unidos: Walt Disney Pictures y Walt Disney Feature Animation.

Disney, W. (productor) y Ferguson, N., Jackson, W., Kinney, J., Luske, H. y Roberts, B. (directores). (1942). Saludos amigos [Cinta cinematográfica]. Estados Unidos: Walt Disney Pictures y RKO Pictures.

Disney, W. (productor) y Geronimi, C., Jackson, W. y Luske, H. (directores). (1951). Alicia en el país de las maravillas [Cinta cinematográfica]. Reino Unido y Estados Unidos: Walt Disney Productions.

Disney, W. (productor) y Geronimi, C., Kackson, W., Kinney, J. y Luske, H. (directores). (1948). Tiempo de melodía [Cinta cinematográfica]. Estados Unidos: Walt Disney Productions.

Disney, W. (productor) y Reitherman, W. (director). (1977). Lo mejor de Winnie the Pooh [Cinta cinematográfica]. Estados Unidos: Walt Disney Pictures.

Eisner, E. W. y Barone, T. (2006). Arts-Based Educational Research. En J. L. Green, G. Camilli y P. B. Elmore (Eds.). Hand book of complementary methods in education research (pp. 95-109). Mahwah: AERA.

Erazo-Jiménez, M. S. (2011). Rigor científico en las prácticas de investigación cualitativa. Ciencia, docencia y tecnología, 20(42), 107-142.

Flecha, R. y Villarejo, B. (2015). Pedagogía Crítica: Un Acercamiento al Derecho Real de la Educación. RIEJS, Revista Internacional de Educación para la Justicia Social, 4(2), 87-100. DOI: http://dx.doi.org/10.15366/riejs2015.4.2

Fullmer, R. (productor) y Dindal, M. (director). (2005). Chicken Little [Cinta cinematográfica]. Estados Unidos: Walt Disney Pictures.

Garabedian, J. (2014). Animating Gender Ro- les: How Disney is Redefining the Modern Princess. James Madison Undergraduate Research Journal, 2(1), 22-25.

Garcés, H. (2000). Investigación científica. Quito-Ecuador: Ediciones Abya-Yala.

García-Lago, V. (2002). ¿Educamos en prejuicios o educamos en valores? Educación y futuro: revista de investigación aplicada y experiencias educativas, 7(4), 1-8.

Giovanni, E. (2007). Disney Films: Reflections of the Other and the Self. Cultura, lenguaje y representación: revista de estudios culturales de la Universidad Jaume I, 4, 91-109.

Gómez, M. T. y Mir, V. (2011). Altas Capacidades en niños y niñas. Detección, identificación e integración en la escuela y en la familia. Madrid: Ediciones Narcea.

Guichot, V. y Bono, C. (2001). De Blancanieves (1937) a Mulán (1998): análisis de los valores, normas y roles sociales transmitidos a través de las películas de Walt Disney. En C. Flecha y M. Núñez (Eds.). La educación de las mujeres: nuevas perspectivas (pp. 45-52). Sevilla: Fundación El Monte.

Guichot-Reina, V. y Merino Delgado, M. A. (2016). Los cortometrajes de animación 
como herramienta didáctica para trabajar la educación en valores en educación infantil. Cuestiones Pedagógicas, 25, 119-132. DOI: http://dx.doi.org/10.12795/ CP.2016.i25.09

Hahn, D. (productor) y Trousdale, G. y Wise, K. (directores). (1996). El jorobado de Notre Dame [Cinta cinematográfica]. Estados Unidos: Walt Disney Pictures y Walt Disney Feature Animation.

Hale, J. (productor) y Berman, T. y Rich, R. (directores). (1985). Taron y el caldero mágico [Cinta cinematográfica]. Estados Unidos: Walt Disney Pictures.

Hernández, R., Fernández, C. y Baptista, P. (2018). Metodología de la investigación. México: Editorial Mc Graw-Hill.

Huerta, R., Alonso-Sanz, A. y Ramon, R. (2019). De película. Cine para educar en diversidad. Valencia: Tirant Humanidades.

Lasseter, J. (productor) y Anderson, S. J. (director). (2007). Descubriendo a los Robinson [Cinta cinematográfica]. Estados Unidos: Walt Disney Animation Studios.

Ley Orgánica 2/2006, de 3 de mayo, de Educación; modificada parcialmente por la Ley Orgánica 8/2013, de 9 de diciembre, para la mejora de la calidad educativa.

López, H. (2011). Detección y evaluación de necesidades educativas especiales en el sistema educativo. Etapa de 0-8 años: escuela Infantil y primer ciclo de Primaria. Revista Educación Inclusiva, 4(1), 11-22.

Lugo-Lugo, C. R. y Bloodsworth-Lugo, M. K. (2009). “Look Out New World, Here We Come?" Race, Racialization, and Sexuality in Four Children's Animated Films by Disney, Pixar, and DreamWorks. Cultural Studies-Critical Methodologies, 9(2), 166-178. DOI: https://doi.org/10.1177/1532708608325937

Marsden, P. (productor) y Leighton, E. y Zondag, R. (directores). (2000). Dinosaurio [Cinta cinematográfica]. Estados Unidos: Walt Disney Pictures.

Martínez, M. (2006). La investigación cualitativa (síntesis conceptual). Revista IIPSI, 9(1), 123-146.

Miñambres, A. y Jové, G. (2000). La atención a las necesidades educativas especiales: de la Educación Infantil a la Universidad. Lleida: Universitat de Lleida.

Monleón, V. (2020a). ¿Y la pedagogía Disney? Trabajando hacia una educación audiovisual de calidad. Artyhum, Monográfico 1: Humanidades Digitales y Pedagogías Culturales, 135-155.

Monleón, V. (2020b). El mantenimiento de una estructura social de clases a través de los largometrajes de la colección "Los clásicos" Disney (1937-2016). Communiars, 3, 76-94.

Muñoz, A. (2000). Hacia una Educación Intercultural: Enfoques y Modelos. Encounters in Theory and History of Education, 1, 81-106.

Napier, S. J. (2001). Confronting master narratives: History as vision in Miyazaki Hayao's cinema of de-assurance. Positions: east asia cultures critique, 9(2), 467-493.

Pappalardo-Robinson, M. (productor) y Glebas, F. (director). (2003). La gran película de Piglet [Cinta cinematográfica]. Estados Unidos: Walt Disney Pictures, Disney Toon Studios y A. Film A/S.

Pentecost, J. (productor) y Gabriel, M. y Golsdberg, M. (directores). (1995). Pocahontas [Cinta cinematográfica]. Estados Unidos: Walt Disney Pictures. 
Monleón, V. (2021). De la normatividad hacia la alteridad. Estudio mixto sobre el tratamiento de la diversidad en películas Disney. Educatio Siglo XXI, 39(3), 83-108.

Ramírez, F. H. y Zwerg-Villegas, A. M. (2012). Metodología de la investigación: más que una receta. AD-minister. Universidad EAFIT, 20, 91-111.

Reguillo, R. (2005). Nosotros y los miedos a la construcción política y cultural de los sentimientos. Argentina: FLACSO.

Roig, B. A. (2012). Educación literaria. Literatura infantil y juvenil. Una propuesta multicultural. Educação, 35(3), 362-370.

Ros, N. (2007). El film Shrek: una posibilidad desde la educación artística para trabajar en la formación docente la lectura de la identidad y los valores. Revista Iberoamericana de Educación, 44(6), 1-12. DOI: https://doi.org/10.35362/rie4462195

Singer, P. (2018). Liberación animal: el clásico definitivo del movimiento animalista. Taurus: Madrid.

Spencer, C. (productor) y Bush, J., Howard, B. y Moore, R. (directores). (2015). Zootrópolis [Cinta cinematográfica]. Estados Unidos: Walt Disney Pictures y Walt Disney Animation Studios.

Spencer, C. (productor) y de Blois, D. y Sanders, C. (directores). (2002). Lilo y Stitch [Cinta cinematográfica]. Estados Unidos: Walt Disney Pictures.

Spencer, C. (productor) y Howard, B. y Williams, C. (directores). (2008). Bolt [Cinta cinematográfica]. Estados Unidos: Walt Disney Pictures y Walt Disney Animation Studios.

Strauss, A. L., y Corbin, J. (2002). Bases de la investigación cualitativa: técnicas y procedimientos para desarrollar la teoría fundamentada. Medellín: Universidad de Antioquia.

Torres, J. (2003). El currículum oculto. Madrid: Morata.

Williams, C. (productor) y Blaise, A. y Walker, R. (directores). (2003). Hermano oso [Cinta cinematográfica]. Estados Unidos: Disney. 